\title{
Caracteristicas y tipologias de la investigación en ciencias sociales: una reflexión sobre la complementariedad de las funciones de transformar y comprender ${ }^{1}$
}

\author{
Nadia Margarita Rodríguez J. ${ }^{2}$ \\ Universidad del Rosario, Bogotá, Colombia ${ }^{3}$ \\ nadia.rodriguez@urosario.edu.co \\ Recibido: 10 de noviembre de 2014 \\ Aceptado: 23 de abril de 2015 \\ Disponible en linea: 30 de noviembre de 2015
}

\begin{abstract}
Se hace referencia a las ciencias sociales en general, pero un mayor énfasis en la sociología, la antropología y la historia, por ser aquellas que con mayor frecuencia combinan y alternan estas dos formas de investigación.

2 Doctora en Ciencias Sociales de lo contemporáneo: Sociología, Antropología, Historia, y Máster en Antropología y Sociología de lo político y del desarrollo, ambos de la Universidad del Panteón de la Sorbona, Paris I. Antropóloga de la Universidad de los Andes. Mis campos de especialidad son la sociología y antropología rural y de las ciencias sociales, aplicadas al desarrollo y a la intervención social. He sido coordinadora de varias investigaciones, semilleros, programas académicos y consultorias.

3 Profesora principal, Universidad del Rosario, investigadora y docente de planta del programa de Sociología de la Escuela de Ciencias Humanas.
\end{abstract}




\title{
Características y tipologias de la investigación en ciencias sociales: una reflexión sobre la complementariedad de las funciones de transformar y comprender
}

\section{Resumen:}

$\mathrm{El}$ artículo discute la aparente oposición que existe entre la investigación aplicada y la académica en las ciencias sociales, con el objetivo de aportar elementos para la articulación de estos dos campos como ejercicios complementarios. Si bien estas dos formas de acercarse al conocimiento y a la intervención sobre lo social determinan diferentes campos profesionales tanto para antropólogos, sociólogos o historiadores, estas formas de investigación no son para nada opuestas. En primer lugar, el artículo intenta mostrar que los argumentos en los que se suelen sustentar las diferencias no son los más interesantes y pertinentes. En segundo lugar, se discuten supuestos y formas de clasificación de la investigación social mostrando que las fronteras no son tan claras y que existen múltiples posibilidades de posicionamiento para los investigadores. Por último, el artículo intenta identificar algunas diferencias que hacen complementarias las funciones de transformación propias a la investigación aplicada y la comprensión de lo social propia a la investigación académica ${ }^{4}$.

Palabras claves: investigación académica; investigación aplicada; tipologias; articulación; ciencias sociales

\section{Characteristics and typologies of social science research: a reflection on the complementarity of transforming and understanding}

\begin{abstract}
The article discusses the apparent opposition between the applied and academic research in the social sciences in order to provide elements for the articulation of these two fields as complementary exercises. Although these two ways of approaching knowledge and social intervention determine different professional fields for anthropologists, sociologists and historians, these forms of research are not really opposed. First, the article attempts to show that the arguments which support the differences are not the most interesting and relevant. Second, assumptions and methods of classification of social research are discussed showing that the boundaries are not so clear and that there are multiple positioning possibilities for researchers. Finally, the article attempts to identify some differences that make complementary the transformation functions of applied research and the social understanding of the academic research.

Keywords: academic research; applied research; typologies; articulation; social sciences
\end{abstract}

\section{Caracteristicas e tipologias da pesquisa em ciências sociais: reflexão sobre a complementariedade das funções de transformar e compreender}

\section{Resumo}

O artigo discute a aparente oposição existente entre pesquisa aplicada y académica nas ciências sociais, com o objetivo de dar elementos para a articulação desses dois campos como exercícios complementares. Bem que estas duas formas de se aproximar do conhecimento e a intervenção sobre o social determinam diferentes campos profissionais tanto para antropólogos e sociólogos quanto historiadores, estas formas de pesquisa não são em nada contrárias. Em primeiro lugar, o artigo tenta demonstrar que os argumentos nos que geralmente se sustentam as diferenças não são os mais interessantes e pertinentes. Em segundo lugar, discutem-se pressupostos e formas de classificação da pesquisa social mostrando que as fronteiras nem são tão claras e que existem múltiplas possibilidades de posicionamento para os pesquisadores. Por fim, o artigo visa identificar algumas diferenças que tornam complementares as funções de transformação próprias da pesquisa aplicada e a compreensão do social própria da pesquisa académica.

Palavras-chave: pesquisa académica; pesquisa aplicada; tipologias; articulação; ciências sociais

4 El documento es una reflexión, producto de mi interés personal por articular las dos formas de investigación social, y parte de mi experiencia como consultora e investigadora universitaria. Además, es producto de una reflexión institucional en torno a un programa de posgrado que combina las dos formas de investigación. No es producto de un proyecto de investigación, por lo que no tuvo una financiación específica. 
De lo que proponemos antes que nada al estudiar la realidad, no se deduce que nosotros renunciemos a mejorarla, estimamos que nuestras investigaciones no merecen ni una hora de pena si ellas solo tuviesen un interés especulativo. Si separamos con cuidado los problemas teóricos de los prácticos, no es para desatender estos últimos: es por el contrario, para prepararnos mejor para resolverlos. (Durkheim, 1987, p. 41)

La identificación de campos diferenciados entre investigación social aplicada (ISAp) y la investigación académica (IA) en ciencias sociales ha venido construyendo fronteras y estereotipos que tienden a situarlas en polos opuestos ${ }^{5}$. Las causas son múltiples y parece pertinente reflexionar sobre esta separación, ya que tiene incidencia en la manera en que nos posicionamos como investigadores y los retos de la formación que impartimos a los profesionales de las disciplinas sociales. Si bien la separación no es nueva ${ }^{6}$, las últimas dos décadas han sido clave para la expansión de unas ciencias sociales enfocadas a diseñar, gestionar y evaluar la intervención social, y adaptadas a campos tan diversos como los estudios de mercados, la publicidad y el trabajo en empresas privadas, en oficinas de personal o de responsabilidad social. Este fenómeno exige una transformación de los campos prácticos y epistemológicos de las disciplinas, y hace pertinente las discusiones sobre los limites y las posturas de los investigadores.

5 El origen de esta separación se sitúa en los esquemas de profesionalización implementados en las instituciones de educación superior. Desde alli se dividen las disciplinas en aplicadas (trabajo social, administración, etc.) y científicas (sociologia, antropología, etc.). Esta separación se entiende como "posturas tradicionales vigentes (entonces y ahora) acerca de la separación entre producción de conocimiento y su aplicación, o entre científicos sociales y planificadores, administradores y profesionales de las ciencias sociales" (Salazar, 1992, p. 15). Algunos autores interesados en el desarrollo de la perspectiva de la investigación aplicada desde hace ya varias décadas (Lewin, 1946; Fals Borda, 1998; Ander-Egg, 2003) han propuesto diferentes aproximaciones para establecer vínculos entre la producción de avances teóricos y cambios sociales al interior de las disciplinas científicas como la antropología y la sociología. Con este punto de partida, nuestra intención es profundizar en esta discusión aportando nuevos elementos a estos debates en el contexto actual de las ciencias sociales.

6 Miriam Jimeno, hablando del ejercicio laboral de los egresados del programa de Antropología, dice: "Tan solo la sede de Bogotá de la Universidad Nacional de Colombia cuenta con mil egresados [...] los profesionales están por todo el país, se adentran hasta lo más remoto de los territorios, en barrios, comunas urbanas, trabajando para organizaciones no gubernamentales, algunos de las propias comunidades, mientras otros lo hacen en instituciones oficiales" (Jimeno, 2007, p. 14). 
El objetivo de este artículo de reflexión es señalar que las dos formas de investigación, aunque no son iguales, son complementarias, y que las ciencias sociales, aun en su vertiente más científica, no dejan de estar ligadas al cambio social, y en su vertiente más aplicada, no se alejan de posturas analíticas propias de cada disciplina. Para ello estructuramos los argumentos en tres partes. En primera instancia, se discuten las ideas que oponen estos campos, para mostrar que los lugares donde se sitúan los límites no son los más adecuados. En segundo lugar, se toman dos clasificaciones o tipologías en las que, a partir del análisis del grado de compromiso de los investigadores, se entrelazan las funciones de comprensión y transformación de las ciencias sociales. Finalmente se exponen las diferentes condiciones en las que se dan estas formas de investigación, señalando las dimensiones prácticas y contextuales que tiene cada una de ellas dentro y fuera de las esferas académicas, resaltando aspectos para tener en cuenta en su articulación.

Esta discusión pretende aportar elementos para el contexto de la investigación social en los ámbitos universitarios en Colombia. Por una parte, considero que permite a los investigadores clarificar nuestras posturas en un contexto universitario en el que se plantean tres funciones: la docencia, la investigación científica y la extensión (como una forma de investigación aplicada). Por otra parte, abre el debate sobre la creación de programas académicos de posgrado con énfasis en investigación científica o en 'profundización'"

Esta es una tendencia que aparece en Colombia en los últimos años; dado el surgimiento en las últimas décadas de programas de posgrado y más recientemente de doctorados, las universidades han optado por diferenciar maestrías orientadas a la investigación, conducentes a la posibilidad de continuar en un doctorado, o bien maestrias que preparan para la vida profesional y que no necesariamente implican una continuidad en el mundo académico. Es el caso de la maestría en Estudios Sociales de la Universidad del Rosario o las maestrias en Geografia, Historia y Antropología de la Universidad de los Andes, entre otras. 


\section{Origen y argumentos de la construcción de dos posturas en la investigación social}

La separación entre la IA y la ISAp se sustenta en representaciones encontradas por parte de quienes defienden una u otra postura. Del lado de la primera se han cuestionado de manera general los procedimientos y resultados de la ISAp, imputándole poca rigurosidad científica y escasa profundidad teórico-metodológica (Salazar, 1992; Fals Borda, 1998). A ello se suma que las intervenciones no se rigen por los mismos estándares y, por lo tanto, no producen resultados que puedan ser debatidos en arenas académicas. Por su parte, la ASAp cuestiona la falta de compromiso de los académicos y la poca efectividad de sus reflexiones en la producción de transformaciones sociales (Ander-Egg, 2003). De allí que la relación entre estas formas de investigación tiende a definirse como una oposición en al menos tres aspectos que analizaremos a continuación: 1) los objetivos de cada postura; 2) el estatus científico de los investigadores, y 3) una serie supuestos establecidos en torno a la articulación de la teoría y la práctica.

\section{La construcción de una oposición a partir de los objetivos}

Lammerink y Wolffers (1998) proponen una primera tipologia para clasificar diferentes formas de investigación social a partir de los objetivos que estas persiguen: la investigación fundamental, la investigación fundamental estratégica y la investigación aplicada. Esta propuesta nos parece relevante porque pone en el centro de la caracterización el alcance de los resultados de la investigación, y de allí se desprende la naturaleza tanto de la postura del investigador como la función que la investigación debe cumplir en relación con la disciplina y la sociedad.

Por una parte, definen la investigación fundamental como aquella que tiene una ambición puramente conceptual y que tiene por objetivo la producción de conocimiento. Esta se aproxima a los hechos sociales a través del análisis de datos producidos por otros o propios, producto de un trabajo de campo empírico. Esta orientación defiende 
la observación externa de los hechos sociales y su traducción en conceptos que permitan dar una o varias explicaciones a los fenómenos. Los aportes de estas investigaciones permiten aumentar el cúmulo de conocimientos sobre la diversidad de los hechos sociales y sus posibles interpretaciones.

En la investigación fundamental se encuentran la mayoría de los trabajos de investigadores y académicos reconocidos que, a través de evidencias científicas crean, nuevos conceptos o discuten aquellos ya validados dentro de los cánones disciplinares ${ }^{8}$. Dentro de estas investigaciones caben las posturas más críticas, que cuestionan las categorias desde las cuales se hacen conceptualizaciones en ciencias sociales, como las de Hayden White (1973) en el caso de la historia o las de Clifford y Marcus (1986) en el caso de la antropología. También recoge las discusiones de corte epistemológico sobre las ciencias sociales y sus objetos de estudio (Wallerstein, 1996; Sperber, 1996; Bosa, 2011).

La segunda es la investigación fundamental estratégica (Lammerink y Wolffers 1998, p. 38), la cual conserva los rasgos de la anterior, pero persigue un objetivo orientado a la transformación indirecta de las situaciones observadas. Se trata de producir conocimientos desde el análisis conceptual de la realidad, pero con incidencia política y estratégica, donde se pueden transformar las situaciones o condiciones en las que están inmersos los grupos sociales estudiados. Sin embargo, enfrenta dos dificultades: las trasformaciones estructurales en la sociedad no se pueden producir de manera inmediata y es necesario pasar por procesos largos y complejos de aprender nuevas dinámicas y desaprender otras, y no es suficiente denunciar o dar a conocer las situaciones críticas, sino proponer formas de solución, partiendo de un estudio serio de los fenómenos sociales'.

8 Un ejemplo pueden ser los conceptos desarrollados por el sociólogo Francés Pierre Bourdieu de Habitus, campo social, violencia simbólica, etc., conceptos que luego son apropiados y discutidos dentro de los ámbitos académicos y aplicados a diversos contextos sociales. Es lo que comúnmente conocemos como investigación académica y se valida con publicaciones científicas.

9 En este caso estarian la mayoria de las investigaciones que se hacen en ciencias sociales, que, aunque no se planteen como un objetivo directo incidir en un aspecto de la realidad, de la política o de una situación determinada de desigualdad, sí contemplan una ambición de traspasar esferas de discusión no académicas. Por eso la tipología se queda corta para clasificar las ciencias sociales. 
Por último, los autores definen la investigación aplicada como aquella que tiene por objetivo transformar, de manera más o menos inmediata, alguna situación social mediante procesos de intervención. En este tipo se incluye una gran diversidad de prácticas que diferenciaremos más adelante. En términos generales, tiene estrategias particulares y rápidas de intervención y objetivos muy puntuales de transformación. En la mayoría de los casos, es una investigación contratada por un 'cliente', que define la meta a alcanzar o la situación a transformar (Burawoy, 2005, p. 204). Estos 'clientes' van desde organizaciones sociales (un sindicato o cooperativa) o empresas privadas, hasta instituciones del Estado, cada una con fines dispares. Los casos aquí son muy amplios y este tipo de investigación es cada vez más generalizada en contextos de Investigación social para el desarrollo. Si bien el propósito es transformar para dar solución a problemáticas sociales, encuentra sus límites en la complejidad de las realidades sociales, que raramente tienen una solución unívoca y pragmática. La crítica más fuerte que se le hace es su carácter paliativo y muchas veces el alcance efimero de sus propuestas.

Esta caracterización por objetivos está en la base de la justificación de la existencia de diferencias -aparentemente insalvables- entre las investigaciones. Sin embargo, la tipología es insuficiente, ya que las investigaciones, en muchos casos, pueden compartir estos tres objetivos de manera simultánea ${ }^{10}$, y una no excluye a la otra. En nuestro ámbito nacional, la investigación en sociología, historia y antropología siempre ha tenido un compromiso con la transformación (Cataño, 1986; Bejarano, 1995; Jimeno; 2007; Rodríguez, 2013). Sin embargo, esta idea de que la diferencia entre ISAp e IA persiguen objetivos diferentes toma fuerza a la hora de clasificar la investigación.

\section{Diferencias en la investigación respecto al estatus cientifico de los investigadores}

En la división tradicional que se presentó anteriormente también existe otro aspecto en el que se ha cimentado la diferencia entre la ISAp

\footnotetext{
${ }^{10}$ Para algunos autores, la investigación social no puede ser tal sin un componente reflexivo y analítico que discuta con los conceptos de las ciencias sociales y sin un componente de búsqueda de las trasformaciones sociales por diversos medios (Ortner, 1994).
} 
y la IA. De acuerdo con los argumentos de autores como Fals Borda (1998) y Ander-Egg (2003), los diferentes ejercicios investigativos no confieren la misma autoridad a los académicos que a los interventores. Los académicos gozan de prestigio y reconocimiento -que se califica según los cánones de la investigación científica con publicaciones y citaciones-, y tienen la posibilidad de situarse críticamente frente a los temas que investigan. Por su parte, los investigadores más comprometidos en los campos de la intervención no cuentan con reconocimiento dentro del ámbito disciplinar.

Tampoco cuentan con el reconocimiento de sus posturas críticas frente a la intervención, como en el caso que narra David Mosse sobre su doble rol como interventor e investigador. En esa experiencia, su postura crítica frente a la intervención fue descalificada tanto por sus colegas en la intervención como por sus colegas académicos. Los unos veían un abuso de su parte al divulgar aspectos de la intervención, mientras que los otros cuestionaban el carácter científico de la misma (Mosse, 2003; 2006). Así, a pesar de proceder de campos profesionales iguales y muchas veces contar con experticias similares, los académicos y los interventores tienden a intentar diferenciarse entre ellos (Uribe y Restrepo, 2000; Giraldo, 2005).

Sin embargo, estos encasillamientos ocultan los distintos grados de compromiso de los investigadores. Existen múltiples ejemplos de comisiones de expertos convocados desde la academia o el sector público para analizar temas coyunturales de política pública o de interés. Es el caso, por ejemplo, del Centro de Memoria Histórica y de las diferentes comisiones de expertos creadas en Colombia y en otros países en contextos de desarrollo y de reparación. Los informes de desarrollo humano son igualmente ejemplos de informes que surgen en ámbitos académicos y generan intervención.

Por otra parte, cabe señalar que sobre los interventores se ha creado la imagen estereotipada del funcionario, más interesado por la eficiencia de los recursos y tiempos. También la de activista político, que se acompaña de la acusación de no sustentar sus acciones en un conocimiento conceptual que le permita intervenir (Castellenet, 2000, 
p. 315). Estas afirmaciones tienen cierto sustento en algunas intervenciones que se hacen de manera poco rigurosa, pero son una minoría y, generalmente, estas no cuentan con científicos sociales. Para el caso colombiano, como ya se señaló, la ciencias sociales han estado comprometidas con la transformación de lo social e incluso han sido muy activas en la critica política ${ }^{11}$, postura que les ha generado -en particular a la sociología- la imagen de disciplina militante (Cataño, 1986; Valencia, 2014). Por otra parte, el hecho de que la carrera académica en ciencias sociales empezara a consolidarse en Colombia solo en la última década, hizo que la mayoría de los egresados de antropología, sociología e historia se orientara a ejercicios profesionales más aplicados e incluso militantes en organizaciones sociales o con grupos indígenas, dentro y fuera de instituciones del gobierno.

Más allá de estas ideas latentes que intentan encasillar el ejercicio de la investigación en ciencias sociales, la separación no parece tan clara en la práctica, y los grados de compromiso de los investigadores dependen de múltiples variables como el contexto, la gravedad de la problemática, o la historia personal de cada uno. En sus investigaciones referidas a los estudios antropológicos sobre el sida en África, Didier Fassin afirma que fue la observación y sensibilización de la gravedad de la epidemia la que llevó a algunos investigadores a comprometerse de manera contundente con la transformación de la salud pública, y no porque este fuera un objetivo de sus investigaciones (Fassin, 1996).

En el caso colombiano, académicos como Orlando Fals Borda, Absalon Machado, Gonzalo Sánchez, Alejandro Reyes, Carlos Gaviría, Jesus Bejarano, Alfredo Molano, Darío Fajardo, María Emma Wills y Luis Jorge Garay, entre muchos otros, son ejemplo de la forma en que los científicos sociales terminan involucrándose en movilizaciones, procesos de transformación, formulación de políticas públicas, investigaciones aplicadas, consultorias para organismos públicos nacionales y extranjeros e. incluso en partidos políticos.

\footnotetext{
${ }^{11}$ Autores como Fals Borda y Absalon Machado en sociologia, Juan Friede y Luis Guillermo Vasco en antropología, o Jesús Antonio Bejarano en historia, son solo los más nombrados de las decenas de académicos que contribuyeron en la consolidación de las ciencias sociales pensadas para la acción y la transformación.
} 


\section{Supuestos sobre la articulación entre teoria y práctica}

Otro punto sobre el cual se sustenta la diferenciación entre la ISAp y la IA es la aproximación que cada una hace de los marcos conceptuales y en relación a la práctica. Autores que se han interesado por reconstruir las tradiciones disciplinares de las ciencias sociales convergen sobre la idea de que en Colombia y América Latina han coexistido las dos posturas, pero en espacios diferenciados (Fals Borda, 1998; Cardoso, 1997, p. 18; Caviedes, 2002; Jimeno, 2007; Correa, 2006) ${ }^{12}$. Esta separación llevó a intentos por diferenciar las prácticas de unos y otros, y de allí nacen los supuestos sobre diferentes aproximaciones con relación a la teoría y la práctica.

Según la diferenciación hecha por Cardoso (1997) para el caso brasileño, la IA parte de exhaustivos marcos teóricos y conceptuales que permiten consolidar nuestro conocimiento sobre lo social. En el plano metodológico, desarrolla amplios estudios empíricos, en los que gran parte de la investigación es destinada al trabajo de campo, a las pesquisas en archivos o al análisis de fuentes secundarias y de datos recabados por otros investigadores. En otros casos, los investigadores se aproximan a los datos y a las investigaciones con propósitos críticos, optando por reflexiones puramente teóricas sobre los trabajos de otros ${ }^{13}$. Esta aproximación ha sido objeto de cuestionamientos por parte de los investigadores de la acción, quienes afirman que esta posición crítica aleja la academia de la realidad y no es tan eficaz a la hora de incidir en la sociedad (Fals Borda, 1988; Castellenet, 2000).

Por su lado, la ISAp no puede desarrollarse sino en relación con los sujetos, más cuando la tendencia participativa se impone en los proyectos y programas de intervención (Rodríguez, 2005). Estas investigaciones se desarrollan en campo y en diálogo con las personas, reto

\footnotetext{
12 Para el caso de la antropología colombiana existieron, durante las décadas del sesenta y setenta, dos espacios en los que se configuraron estas diferencias. Antropólogos académicos desarrollando investigaciones científicas en los nacientes programas de Antropolgía de la Universidad Nacional y de los Andes o el Instituto de Etnografia, y los antropólogos funcionarios, que Correa denomina como la corriente del 'indigenismo estatal' (Correa, 2006, p.18), en la oficina de asuntos indígenas del Ministerio del Interior (Rodríguez, 2013, p. 226).

${ }^{13}$ Ver discusiones sobre las posturas de la sociología crítica en la presentación en video de Didier Fassin (2009).
} 
interesante que requiere no solo producir conocimiento sino, además, hacerlo con los actores, construir propuestas a partir de una relación que está cargada de intereses y representaciones que favorecen o limitan la intervención.

Estas condiciones que acompañan a una y otra forma de investigación crean la idea de que la fortaleza de la IA radica en la teorización alejada de la práctica, y a la inversa, que la ISAp se sustenta en la práctica con una débil base teórica (Fals Borda, 1998; Fassin 2009).

Se trata de ideas que reflejan una verdad 'a medias', ya que, efectivamente, los objetivos y las ambiciones de ambas perspectivas están centrados en lugares diferentes, que hacen que se aproximen de manera distinta a las dos facetas de la investigación. Considero que ambas, teoría y práctica, son constitutivas de todas las aproximaciones de los científicos sociales. El sustento empírico de la IA está presente no solo en los trabajos de campo o de archivo, sino en su capacidad de incidencia en la práctica. Por una parte, haciendo visibles estructuras y situaciones que son problemáticas, como por ejemplo, formas de dominación o discriminación que pueden ser naturalizadas. En segundo lugar, el análisis que propone la IA busca echar luz sobre ciertas estructuras y las categoriza, desplazando así nociones asentadas en el sentido común (Bosa, 2011). Además, la IA, incluso la más centrada en la crítica, ha jugado un papel fundamental en la denuncia y reflexión de desigualdades sociales como el género, la raza o la clase social y, al explicarlas como construcciones, ha permitido la transformación de ciertas prácticas para aceptar las diferencias en la sociedad (Fassin, 2009).

Por su parte, la ISAp, aunque no en todos los casos, también ha analizado rigurosamente la realidad social con marcos conceptuales, estudiando políticas públicas, evaluando impactos sociales y diseñando dispositivos de intervención. Los ejemplos son múltiples pero, solo por citar uno, el equipo de consultores del Programa de las Naciones Unidas para el Desarrollo, que realizó el Informe de Desarrollo humano 2011, estuvo compuesto por los académicos más sobresalientes sobre los problemas agrarios en Colombia 
(PNUD, 2011). Por otra parte, los marcos metodológicos que se aplican se desprenden de investigaciones teóricas, por ejemplo, el marco lógico, instrumento clave de los organismos internacionales de cooperación, fue concebido a partir de teorias de análisis sistémico (Comisión Europea, 2002, p. 78). Así, existe un creciente interés por vincular la IA y sus procedimientos a la intervención, y esto se ve reflejado en dos aspectos: el primero es que la mayoría de egresados de las facultades de ciencias sociales laboran en campos aplicados y son cada vez más buscados por empresas públicas y privadas. Pero ocurre que los egresados, en el caso colombiano, han sido formados más para la IA que para la intervención (Uribe, 2005). El segundo aspecto en el que se evidencia este acercamiento es la creciente labor de extensión que se hace en las universidades a través de consultorías, en las que los académicos participan en dispositivos de intervención pública y privada.

Con esto, resulta dificil determinar la justa mezcla entre teoria y práctica en la investigación en ciencias sociales social. Los ejemplos en los polos opuestos entre una investigación puramente teórica y una puramente práctica no parecen frecuentes. Sin duda, en el plano de las prácticas, los repertorios de acción de los investigadores son distintos y el hecho de que los investigadores muchas veces no se reconozcan como pares es significativo. Sin embargo, existen aspectos que nos permiten pensar que no se trata de campos epistemológicos completamente apartados y que necesariamente debe existir un diálogo entre las dos posturas. Constatación de lo anterior es que son los egresados de las mismas disciplinas quienes ejercen en ambos campos, todos han recibido las mismas herramientas conceptuales y metodológicas y comparten formas de entender lo social. Se podría afirmar, incluso que tienen en común un habitus y que pertenecen a un mismo campo social (Bourdieu, 1984). Por otra parte, sus prácticas son compartidas, lo cual permite pensar que, aunque con objetivos diferentes, se enfrentan a los mismos problemas de maneras similares. Este punto permite resaltar la complementariedad de las dos posturas y el interés de diálogo entre ellas. Existen experiencias exitosas que demuestran 
esta compatibilidad, como es el caso de algunos institutos de investigación que incorporan procesos de intervención ${ }^{14}$.

\section{Propuestas para salir de los esquemas excluyentes entre la IAy la ISAp}

Diferente a las tipologías que hemos presentado en la primera parte, donde se resaltan las diferencias en la investigación en ciencias sociales, Didier Fassin $(1996 ; 1998)$ propone relacionar los campos aplicado y académico a partir de una reflexión sobre los grados de implicación de los investigadores en relación con la sociedad o con los problemas investigados. Para ello, el autor retoma a Norbert Elias (1993), afirmando que los investigadores se sitúan frente a sus objetos de estudio dentro de un eje que va del compromiso a la distancia. Posturas antagónicas, pero que pueden ayudar a discernir las aproximaciones científicas a sus objetos. Estos dos polos propuestos por Elias representan también las posturas de los investigadores; mientras la posición comprometida privilegia una postura política y militante, la distancia privilegia una postura reflexiva y critica. Estas posturas permiten identificar "las relaciones de los investigadores hacia sus objetos de investigación y hacia la sociedad en sí misma ${ }^{15}$ " (Fassin, 1998, p. 45).

De acuerdo con este criterio, la investigación más comprometida sería la aplicada y la investigación más distanciada sería la académica (Fassin, 1998). Sin embargo, el esquema lineal de Elias no convence del todo a Fassin, quien comprende las posiciones de compromiso y distancia como dos ejes de un plano cartesiano en el que sitúa a los investigadores en distintos grados de acercamiento a uno u otro eje. El eje horizontal, representando la perspectiva en la que el investigador

${ }^{14}$ Es el caso del Centro de Investigación y Educación Popular en Bogotá (CINEP) y El Centro de Memoria Histórica. También es el caso de múltiples centros de investigación universitarios que, a pesar de estar inmersos en contextos académicos, cada vez más desarrollan consultorias para actores públicos y privados, nacionales e internacionales. Es el caso del Centro de Estudios Sociales (CES) de la Universidad Nacional o del Centro Interdisciplinario de Estudios sobre Desarrollo (CIDER) de la Universidad de los Andes, entre otros.

${ }^{15}$ La traducción del francés es mía. 
establece una distancia con el objeto de estudio en la cual se fortalece su capacidad critica por la distancia, y el eje vertical, el grado de compromiso del investigador que, por tanto, determinaría su grado de acción implicada en relación con la población del estudio.

A partir de las diferentes aproximaciones de los investigadores, Fassin termina por proponer una tipología tripartita. Por una parte, habria una investigación aplicada que estaría en un punto medio, tanto del eje horizontal como del vertical, es decir, que se acerca de la misma manera a la acción y al análisis crítico. Un segundo tipo, la investigación crítica sería aquella que toma distancia en términos de la acción, para situarse en una posición en extremo analítica. Y, por último, una investigación implicada, que sería aquella que se sitúa más cerca a la acción comprometida y que se distancia del análisis crítico (Fassin, 1998, p. 54).

Para sustentar este sistema de posiciones, Fassin vuelve a un postulado de Elias, afirmando que:

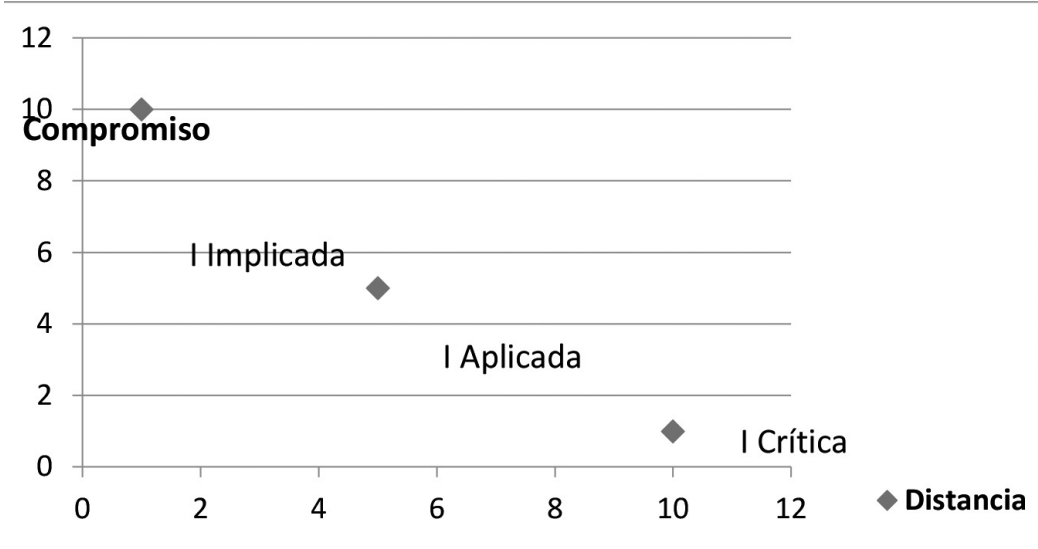

Gráfico 1. Relación entre compromiso y distancia crítica Fuente: elaboración propia 
La tesis de Elias de la indisociabilidad de las dimensiones epistemológicas y políticas [...] permiten sobrepasar la ilusión positivista. [...] El investigador está comprometido con respecto al mundo que estudia a la vez como sujeto que conoce que como sujeto ciudadano. [...] Situando el compromiso y el distanciamiento como dos polos inseparables y conflictivos de la actividad humana, él nos está invitando a pensarlos juntos. (Fassin, 1998, p. 43)

En este sentido, la relación que los investigadores desarrollan con respecto a sus problemas de estudio tiene mucho que ver con el hecho de clasificarse en una u otra postura. Para la reflexión que intentamos construir sobre las relaciones entre ISAp e IA, este problema de la distancia e implicación parece clave paraa entender cómo una no excluye a la otra.

Otra propuesta es la sociología pública de Michael Burawoy (2005), que viene acompañada de otra tipología que permite pensar los roles de la investigación en términos de implicación y reflexión ${ }^{16}$. El sociólogo propone un sistema con dos ejes, uno que habla sobre el tipo de conocimiento que se produce desde la disciplina y otro sobre el tipo de audiencia al que está dirigido. El primer eje identifica la producción de un conocimiento instrumental y otro más reflexivo. El segundo eje identifica una audiencia académica y otra extra académica. Al realizar una tabla con estas variables, se producen cuatro tipos de aproximación sociológica: una sociología profesional, una crítica, una práctica y una pública -que es la que Burawoy intenta institucionalizar- (Tabla 1).

\begin{tabular}{|l|l|l|}
\hline \multicolumn{1}{|c|}{ AUDIENCIA } & \multicolumn{1}{c|}{ ACADÉMICA } & \multicolumn{1}{c|}{ EXTRA ACADÉMICA } \\
\hline Instrumental & & \\
\hline Reflexivo & Profesional & Práctica \\
\hline
\end{tabular}

Tabla 1. Propuesta de la sociología pública Fuente: Burawoy (2005, p. 206)

${ }^{16}$ Este debate surge cuando Burawoy es presidente de la American Sociological Association y, en sus términos, la sociología atraviesa una crisis, viéndose obligada a repensar sus paradigmas y objetos. 
Esta nueva clasificación que nos propone cuatro aproximaciones o posturas de los investigadores frente a sus investigaciones mostraría una "división del trabajo sociológico", en términos del autor (Burawoy, 2005, p. 2001). La profesional (también traducida como académica) desarrolla conocimientos que permiten la construcción y comprensión de los fenómenos que estudia. La crítica se encarga de cuestionar y analizar esos conocimientos que se producen sobre lo social y a la disciplina misma. Una sociología práctica (o aplicada) produce conocimientos instrumentales que sirven para la transformación de lo social $\mathrm{y}$, finalmente, una sociología pública pretende establecer unas posiciones analíticas y reflexivas sobre esos procesos de transformación de lo social, contribuyendo a un posicionamiento de los investigadores frente a la realidad y su trasformación.

Si las dos primeras están inscritas en un ámbito académico donde se desarrollan y al cual se dirigen, las dos últimas estan destinadas a tener un impacto en la sociedad, más allá del mundo académico. La tipología surge porque la sociología crítica y la práctica parecen haber tomado la delantera, y el planteamiento de Burawoy pretende fortalecer una postura intermedia entre las dos; por una parte, salvándose de la visión puramente instrumental a través de una sociología más implicada y a la vez crítica, orientada a salir del ámbito académico, $y$, por otra, incitando a los investigadores a posicionarse frente a sus audiencias. Esta propuesta resulta muy interesante, al incorporar las variables de la población de destino de la producción científica.

Tanto la propuesta de Fassin como la de Burawoy tienen puntos en común que aportan argumentos a esta reflexión. Por una parte, las dos son válidas para el conjunto de las ciencias sociales, aunque la primera fue pensada para el caso de la antropología y la segunda para el caso de la sociología ${ }^{17}$. Las dos toman la relación de los investigadores con los actores sociales o los problemas que estudian como eje articulador de la construcción de los tipos. Además, retoman la problemática del compromiso, muy relevante en esta discusión, pues cualquiera que sea el objetivo de un investigador, este adquiere com-

${ }^{17}$ En la antropología también existe una vertiente pública liderada por Robert Borofsky (2002), que propone, de manera similar a Burawoy, reorientar la disciplina sacándola de los ámbitos académicos para discutir las posturas analiticas con la sociedad civil. 
promisos y responsabilidades con las poblaciones o grupos a los que estudia. Tanto en el planteamiento de Fassin como en el de Burawoy se hace explicita la complementariedad y en ello justifican la pertinencia de las ciencias sociales. Para Burawoy, la sociología que permite unir los dos extremos es la pública, capaz de constituir un debate crítico y político sobre la realidad social. Por su parte, Fassin lo resuelve proponiendo una investigación aplicada que esté en un término medio entre el compromiso y la distancia analítica, es decir, una intervención que no sea puramente instrumental.

En las dos tipologías también está la idea de indisociabilidad, que está en el centro de la propuesta de Elias utilizada por Fassin y que es importante en el argumento de Burawoy, cuando afirma que estas corrientes constituyen una división del trabajo sociológico -retomando a Durkheim- y que por lo tanto establecen un "lazo orgánico", en el que ninguna puede sobrevivir en desmedro de las otras y en la que cada una le da a la otra una fortaleza para afrontar las debilidades (Burawoy, 2005, pp. 205-206). Otro de los aspectos interesantes de las dos tipologías es el carácter temporal y móvil que tienen los investigadores dentro de una dimensión u otra. A lo largo de su carrera, el científico social oscila entre momentos en que su actividad es más política -cuando se encuentra en campo y tiene una relación más estrecha con los actores de su estudio, que a la vez esperan de él una serie de actitudes implicadas- $y$, en momentos, de mayor distancia, necesarios para construir una visión reflexiva de lo estudiado. Sin que una posición afecte más o menos en su vida profesional, parecerían ser momentos por los cuales todo investigador debe pasar. No significa que los investigadores simplemente oscilen entre un lado y otro, pues la implicación normalmente permanece, aunque los objetivos que se persigan sean más académicos o políticos (Cardoso, 1997).

Las tipologías nos llevan a reflexionar sobre las formas de mantener el compromiso político o moral en los momentos de distanciamiento y cómo seguir siendo críticos en los momentos de mayor implicación. Para Burawoy, la sociología pública tendría esta misión, con una separación en dos sub ramas, una tradicional y otra orgánica. La tradicional es aquella que lleva a la escena pública los debates sociales que son 
problemáticos: "el sociólogo público tradicional instiga debates dentro de o entre los públicos [...]" (Burawoy, 2005, p. 202). Por su parte, la orgánica trabaja en conexión con un público que se hace visible en la sociedad gracias a la intervención del sociólogo. Su propósito es 'hacer visible lo invisible'. Existen casos para los cuales es más fácil establecer una relación entre la dimensión política y la reflexiva. La academia colombiana, en sus orígenes, presenta rasgos de lo que Burawoy llama sociología pública, y varios de los pioneros de la sociología y la antropología nacional podrían clasificarse tanto en la vertiente tradicional como en la orgánica (Giraldo, 2005; Jimeno, 2007).

La propuesta de Fassin aporta un elemento interesante en su uso de las categorias de compromiso y distancia. Estas se presentan como cualidades donde no hay medidas ni límites; se trata de condiciones permanentes. En su esquema, en un plano cartesiano es muy fácil entender la ausencia de fronteras y la presencia única de puntos en los cuales se articulan las dos posiciones. A pesar de que Fassin también cae en la tentación de establecer categorías, para nosotros la imagen resulta más interesante sin ellas.

El problema de las tipologías es la tentación de clasificar o clasificarse en alguno de los tipos. La institución de una sociología pública implica necesariamente construir una separación. En este sentido, esta perspectiva se completa con la idea de compromiso, que parece más acertada que la de posicionamiento. Se trata más de una reflexión sobre la utilidad de la investigación que de los públicos a los que esta se orienta. Cuando Fassin propone dos ejes, está pensando en ejes constitutivos e ineludibles sobre los cuales se tiene una responsabilidad, adquirida al entrar en el campo de las ciencias sociales. Esta imagen refleja bien la ambición de Burawoy de transformación y comprensión como rasgos inseparables de las ciencias sociales, como ya lo eran cuando Durkheim, Weber o el mismo Compte estaban pensando en la constitución de una ciencia de lo social. 


\section{Aspectos a tener en cuenta para articular la IA y la ISAp}

$\mathrm{Si}$, finalmente, no existe una división excluyente entre IA y la ISAp, como hemos intentado mostrar, y si los investigadores pueden optar por múltiples posturas, es conveniente terminar esta reflexión aportando elementos sobre las implicaciones que tiene el tránsito entre las diferentes posibilidades de investigación social. Consideramos que este puede ser un aporte relevante para los jóvenes científicos sociales en el contexto actual, en el que las prácticas profesionales, tanto en la academia como fuera de ella, implican nuevos retos para acceder a recursos para la investigación y para la intervención.

Conviene en este punto aclarar que no toda la ISAp es igual y que, para nuestra argumentación, hay algunas formas de esta que no pueden dialogar de la misma manera con la investigación académica. Para ello, haremos una clasificación diferenciando tres tipos de ISAp. La primera es aquella que se caracteriza por su compromiso con el mejoramiento de las condiciones de vida de las personas desde una mirada crítica, como la investigación acción y la investigación acción participativa (IAP), donde se encuentran autores como Pretty (2000, p. 35), Fals Borda (1998), Max Neef (1993) y Barbier y Lemery (2006). La segunda es aquella que se realiza en el contexto de los programas y politicas que buscan promover el desarrollo, y persigue el objetivo de mejorar las condiciones de vida para facilitar la integración de las sociedades marginadas a la sociedad en general. En esta categoría se encuentran las intervenciones realizadas por agencias de cooperación internacional, instituciones públicas y las intervenciones gestionadas por ONGs. La tercera está constituida por las intervenciones que ocurren en empresas privadas con fines privados, como agencias publicitarias, consultoras que hacen estudios de todo tipo $u$ oficinas de responsabilidad social y de gestión humana al interior de las empresas. Es claro que algunos de los estudios en estos contextos se alejan de las ambiciones de las ciencias sociales, pero ciertamente son un nicho cada vez más robusto de empleo para los egresados de disciplinas como la antropología y la sociología. Los objetivos allí son variados, pero por lo general buscan favorecer los beneficios de la empresa. 
Es aquí donde los métodos de las ciencias sociales son muy útiles, pero los objetivos pueden llegar a tornarse antagónicos (Paz, Saenz, Camelo, y Muñoz, 2010). Esta es la intervención más distante de los ámbitos académicos, pero es importante reflexionar sobre las implicaciones de este ejercicio profesional en el seno de las ciencias sociales.

La primera implicación que tiene este tránsito entre la IA y la ISAp tiene que ver con la autonomía de la investigación, que está marcada por el acceso a recursos de financiación y por la pertinencia de los temas y la manera como estos se definen. En cuanto a los recursos materiales, cuando hacemos ISAp se suele contar con medios de financiación, pero estos están comprometidos para ciertos temas y en tiempos determinados, que se definen con base en agendas politicas, económicas, sociales o humanitarias. Ocurre de manera distinta en la IA, donde casi siempre el investigador define la problemática que le interesa, sus métodos y marcos de análisis, la financiación suele ser escasa y muy competida con base en criterios de calidad. Esta es una diferencia que los investigadores han aprendido a utilizar para circular entre las dos posturas, ya que una ISAp o consultoria, puntualmente, se puede convertir en un medio para financiar parte de una IA, o puede servir para visibilizar resultados de largas trayectorias investigativas que, sin ese apoyo, no tendrian una visibilidad fuera de las audiencias académicas. En este sentido, es interesante pensar en la complementariedad de los campos.

Esta autonomía económica y temática de la IA le permite cuestionar más libremente las categorías y estructuras en la sociedad. Por ello, es una diferencia a tener en cuenta y, en lo posible, no se puede perder en el ejercicio profesional. Si bien en la ISAp el organismo que la contrata impone ciertos límites (temporales, estratégicos y conceptuales), es importante que no llegue a limitar los hallazgos. Esto genera un reto importante para los científicos sociales; mantener una postura crítica desde adentro. El caso de David Mosse, que ya hemos mencionado, resulta interesante para entender que, en las intervenciones para el desarrollo, no siempre es bien recibida la postura reflexiva (Mosse, 2003; Rodríguez, 2013). 
Otro aspecto relevante a tener en cuenta es la temporalidad; algunas intervenciones, y sobre todo aquellas que se hacen en contextos de proyectos de promoción del desarrollo en consultorías, muy pocas veces son de largo alcance. Incluso, pueden llegar a ser de unos pocos meses-, lo cual claramente es insuficiente para generar una transformación. La IA y la ISAp que se hacen en contextos de mayor compromiso, por el contrario, tienen agendas de larga duración en las que varios años son necesarios para investigar un problema y, por lo general, estas investigaciones se suscriben en temas que los investigadores trabajan a lo largo de toda su carrera.

Este es otro de los aspectos por los cuales en el ejercicio contemporáneo de la investigación social se establecen relaciones entre la IA y la ISAp, ya que muchas intervenciones y consultorias requieren grados de experticia para los cuales solo los académicos tienen la suficiente experiencia. Es por eso que, hoy en día, las universidades también son nichos de producción de investigación aplicada que se incorporan bajo la denominación de servicios de 'extensión universitaria'. Ahora bien, otro aspecto a tener en cuenta es que los compromisos frente a las personas con quienes se hace IA e ISAp tampoco son los mismos. Las lógicas de la intervención hoy exigen la participación de los actores involucrados y, aunque este principio resulta utópico y muchas veces es simbólico, implica un reto importante que no necesariamente es una preocupación primordial de la IA. La participación supone la toma de decisiones de manera colectiva en todas las etapas de una intervención, lo que implica implícitamente compartir el poder. Según A. Meister:

[...] la participación realiza una distribución del poder, incluso aunque no se trate de una distribución equitativa, algo que sucede muy raramente en las relaciones sociales [...] Por esto, hablar de fomentar la participación no es solamente formar a los individuos y animarlos a emprender iniciativas, sino sobre todo estar dispuesto a compartir el poder con esas personas [...] En este sentido, la mayoría de los programas tienden a velar este aspecto de la participación. (Meister, 1977, p. 63) 
Es así como, por lo general, la participación se entiende como procesos de consulta con las comunidades, sin reflexionar específicamente alrededor del tipo de participación que se quiere fomentar. En más de un dispositivo, los indicadores de participación son las listas de asistencia, lo cual dista mucho de los propósitos de involucrar realmente a las comunidades en la intervención. Esta característica es problemática, ya que implica negociar con múltiples actores que tienen intereses particulares, limitando así la autonomía de las investigaciones. La producción de falsos consensos sociales genera dificultades importantes en la producción de conocimiento y de cambio en la intervención social. Así, mientras la ISAp adquiere un compromiso frente a los sujetos de la intervención que se ve limitada por ese mismo compromiso, la IA puede asumir una actitud más crítica para dar cuenta de lo problemático de estas estructuras ${ }^{18}$.

\section{Comentarios finales}

Hemos desarrollado una argumentación mostrando que la investigación académica y la investigación social aplicada no son polos opuestas ni excluyentes la una de la otra. Para ello, partimos de discutir algunas oposiciones que han sustentado la idea de que se trata de dos campos diferenciados en las ciencias sociales. Mostramos que tanto por los objetivos que persiguen como por las representaciones relacionadas con el estatus de los investigadores o las relaciones entre teoría y práctica, no existe una verdadera oposición. Los objetivos de transformar y comprender son perfectamente complementarios en el ethos disciplinar de las ciencias sociales, puesto que se encuentran en sus orígenes y también en las prácticas cotidianas de los investigadores. Resaltamos que, al no existir una oposición entre teoría y práctica, tampoco existen ciencias puramente analíticas y otras puramente empíricas, sino maneras diferentes de abordar esta relación.

\footnotetext{
${ }^{18}$ En un caso concreto de estudio en el que participamos en una intervención y posteriormente en una investigación académica que buscaba analizar esa misma intervención, solo a partir del análisis reflexivo pudimos entender las estrategias de algunos de los participantes en el proyecto, que facilitaron o limitaron los resultados de la intervención (Rodriguez, 2005; 2013).
} 
En un segundo momento, con la ayuda de las tipologías de Fassin y Burawoy, tomamos elementos para reflexionar sobre los múltiples posicionamientos posibles con respecto al compromiso, la reflexividad y las audiencias a las que se dirige la investigación en las ciencias sociales. Pero sobre todo intentamos aportar a esta discusión resaltando la idea de que los profesionales de las ciencias sociales transitan en distintos niveles de esas variables, sin necesidad de crear encasillamientos en su ejercicio profesional.

Tratamos, por último, de señalar aspectos relevantes a tener en cuenta para el ejercicio tanto de la IA como de la ISAp que, lejos de oponerlas, proponen reflexiones interesantes sobre el quehacer de los científicos sociales. Por una parte, hay consideraciones materiales y temporales que deben ser tenidas en cuenta a la hora de complementar la una con la otra; implicaciones en cuanto al manejo de los datos, su divulgación y la perspectiva crítica para uno y otro ejercicio. También hablamos de los retos, implicaciones y compromisos que se adquieren con las poblaciones con las que trabajamos y, finalmente, abordamos las ventajas que tiene el hecho de poder circular entre estas formas de investigación y las sinergias que se producen cuando aprovechamos las múltiples posibilidades de posicionarse al hacer investigación.

Esta demostración nos permite concluir con una reflexión sobre las funciones de la investigación social, la posición del investigador y el significado del compromiso en las ciencias sociales.

En cuanto a las funciones, hemos demostrado que desde sus orígenes las ciencias sociales han tenido una ambición transformadora, y cabe señalar que los casos de las investigaciones que hemos citado a lo largo del texto, tanto desde la ISAp como en la IA, siguen estando comprometidas con el cambio social, el mejoramiento de las situaciones de desigualdad, exclusión, dominación, la construcción de procesos de reparación y, en general, propenden por generar bienestar social. 
En cuanto al compromiso de los investigadores y la manera en que estos se aproximan a los problemas que estudian, vimos que en distintos momentos es posible situarse en diversas posturas que les permiten articular reflexividad y compromiso. Lo interesante es saber que existe una diversidad de posibilidades abiertas y la formación de los profesionales en ciencias sociales abre estas posibilidades. Sin duda, es una reflexión interesante en los marcos universitarios e institucionales donde se produce conocimiento y se forman científicos sociales, pues los currículos y las prácticas deberían pensarse en función de ampliar la perspectiva de esta gran diversidad profesional a los estudiantes o jóvenes investigadores, no solo para que puedan enfrentar profesionalmente todos los retos que afrontan, sino además para que tengan las herramientas para elegir dónde quieren posicionarse. De todas formas, en su vida profesional, lo más frecuente es que se vean abocados a desempeñarse en campos aplicados, que es donde se encuentran la mayoría de los egresados de las carreras de antropología y sociologia actualmente. Por otra parte, si nos fijamos en la trayectoria de los investigadores que se encuentran en los ámbitos académicos, muy probablemente encontraremos un número significativo de trabajos aplicados.

En cuanto al compromiso, las tipologías que hemos citado nos han hecho pensar en la relación que establecen los investigadores con sus campos de estudio y las personas con las que interactúa en él, en relación con sus grados de compromiso y distancia y las posiciones políticas y críticas, encontrando allí una vía interesante para pensar la articulación de los objetivos de transformación y comprensión de las ciencias sociales. De igual forma, una pregunta interesante que surge al respecto es si queremos -o se necesitan- unas ciencias sociales comprometidas con el cambio social. En este sentido, si los paradigmas de la objetividad científica han sido ya ampliamente cuestionados, ¿por qué seguir pensando que la posición crítica o distanciada es la más científica? Estas preguntas parecen haber sido superadas, pero creemos que están en el fondo de las divisiones que aún hoy persisten entre los investigadores. 
En el caso particular de Colombia, las ciencias sociales estuvieron marcadas por un fuerte compromiso de cambio y eso, hoy más que nunca, lo vemos reflejado en las posiciones que toma la academia frente a problemáticas como las negociaciones de paz, la reparación a las víctimas y la reconstrucción del tejido social en el posconflicto. En esa misma medida, ahora que la IA y la aproximación científica se empieza a desarrollar con mayor fuerza y calidad en el país, en el seno de universidades públicas y privadas, estas disciplinas han podido posicionarse mejor mediante la creación de un sinnúmero de programas nuevos de pregrado, maestría y doctorado, sin perder la capacidad de compromiso al tiempo que se hacen más reflexivas (Restrepo, 2007; Uribe, 2005; Jimeno, 2007). Por ello, está reflexión invita a abrir esta discusión sobre cuáles son las ciencias sociales que necesita nuestra sociedad y cuáles son los múltiples roles que estas pueden desempeñar desde la academia y desde otros lugares, donde cada vez tienen mayor presencia los científicos sociales.

\section{Referencias}

Ander-Egg, E. (2003). Repensando la investigación-acción-participativa. Argentina: Lumen-Humanitas.

Barbier, M. Chia, E. Lemery B. (2006). La Recherche-Action en Pratique: Réflexions autour d'une étude de cas. Documento de trabajo no publicado. Observatorio TERA. CIRAD.

Bejarano, J. A. (1995). Una agenda para la paz: Aproximaciones desde la teoría de la resolución de conflictos. Bogotá: TM Editores.

Borofsky, R. (2002). The four subfields: Anthropologists as mythmakers. American Anthropologist, 104(2), 463-480.

Bosa, B. (2011). Des concepts et des faits La double contradiction des sciences sociales. Revue Labyrinthe, (N 37), 121-147. 
Bourdieu, P. (1984). Questions de Sociologie. Paris: Editions de Minuit.

Burawoy, M. (2005). Por una sociología pública. Politica y Sociedad, 42(1), 197-225.

Castellanet, C. (2000). Le PAET en Amazonie brèsilienne: diagnostic et participation paysanne dans un programme de recherche-action négociée. En Lavigne Delville, Sellama, N. E., y Mathieu, M. Les enquêtes participatives en débat (pp. 293-326). Paris: Editions Gret, Kartala, Icra.

Cataño, G. (1986). La sociología en Colombia. Bogotá: Plaza y Janés.

Cardoso, F. (1997). Conocimiento y práctica política. En Fals Borda (Ed.). Participación popular: retos del Futuro (pp. 11 -19). Bogotá: ICFES, IEPRI, Colciencias.

Caviedes, M. (2002). Solidarios frente a colaborativos: antropología y movimiento indígena en el Cauca en las décadas de 1970 y 1980. Revista Colombiana de Antropología, 38, 237-260.

Clifford, J. y Marcus, G. (Eds.). (1986). Writing Culture: the Poetics and Politics of Ethnography. Berkeley: University of California Press.

Comisión Europea - EuropeAid (2002). Materiales del curso: El enfoque del marco lógico. Manual de Gestión del Ciclo de un Proyecto.

Correa, F. (2006). Interpretaciones antropológicas sobre lo "indígena". Universitas Humanistica, (62), 15-41.

Durkheim, E. (1987/1893). La división social del trabajo. Madrid: Ediciones Akal.

Elias, N. (1983/1993). Engagement et distanciation. Paris: Fayard.

Escobar, A. (1998). La invención del tercer Mundo: construcción y deconstrucción del desarrollo. Bogotá: Editorial Norma.

Fals Borda, O. (1988). Knowledge and people's power. Delhi: Indian Social Institute. 
Fals Borda, O. (1998). Participación popular: retos del Futuro. Bogotá: ICFES, IEPRI, Colciencias.

Fassin, D. (1996). L'anthropologíe dans les strategies de lutte contre le SIDA. En Salomon y Toubon (Eds.). Sida, sociétés et populations (pp. 246-257). Paris: Ed John Libbey.

Fassin, D. (1998). L'anthropologie entre engagement et distanciation. Essai de sociologie des recherches en sciences sociales sur le sida en Afrique. En Becker, Dozon, Obbo y Toure (Eds.). Vivre et penser le SIDA en Afrique (pp. 538-615). Paris: Ed Karthala, IRD.

Fassin, D. (2009). Les sciences sociales critiques peuvent-elles être utiles? Ciclo de conferencias "A quoi servent les sciences humaines". Revista Tracés. Recuperado de http://www.dailymotion.com/video/x9pygm_une-sciencesociale-critique-peutel_news.

Giraldo, P. (2005). Adiós a la inocencia: crónica de una visita al estilo nacional de hacer antropología. Revista Antípoda, (1),185-199.

Jimeno, M. (2007). Naciocentrismo: tensiones y configuración de estilos en la antropología sociocultural colombiana. Revista Colombiana de antropología, $43,9-32$

Lammerink, M. y Wolffers, I. (1998). Aproches participatives pour le développement durable. Paris: Editorial Kartala, IPD.

Lewin, K. (1946). Action Research and Minority Problems. Journal of Social lssues, 2 (4), 34-46.

Max Neef, M. (1993). El desarrollo a escala humana: Conceptos, aplicaciones y algunas reflexiones. Barcelona: Editorial Nordam-comunidad e Icaria.

Meister, A. (1977). Participation pour le développement. Paris: Ed économie et humanisme: Les éditions ouvrières. 
Mosse, D. (2003). The rule of water: Statecraft, Ecology and Collective Action in South India. Nueva Delhi: Oxford University Press.

Mosse, D. (2006). Anti- social Anthropology? Objectivity, objection, and the ethnography of public policy and professional communities. Journal of the Royal Anthropological Institute, 12, 935-956.

Ortner, S. (1994). Theory in Anthropology since the Sixties. Comparative Studies in Society and History, 126(1), 126-166.

Paz, A., Saenz, J., Camelo, V., y Muñoz, N. (2010). ¿Cómo se transforma lo social? Discursos y prácticas de intervención en Cali. Santiago de Cali: Universidad ICESI.

Pretty, J. (2000). Des systèmes de recherche alternatifs pour une agriculture durable. En Lavigne Delville, Sellama, N. E., y Mathieu, M. (Eds.). Les enquêtes participatives en débat (pp. 29-54). Paris: Editorial Gret, Kartala, Icra.

Programa de las Naciones Unidas para el Desarrollo. PNUD (2011). Colombia rural. Razones para la esperanza. Bogotá: Ed PNUD.

Restrepo, G. (2007). La sociología colombiana en la víspera de sus cincuenta años. Revista Colombiana de Sociología, 28, 9-12.

Rist, G. (1996). Le développement: Histoire d'une croyance occidentale. Paris: Editorial Presses de sciences po.

Rodríguez, N. M. (2005). Caracterización y diagnóstico de las organizaciones campesinas de la región Huetar Norte: una propuesta de fortalecimiento a través de la participación. En Samper (Comp.) Trayectorias y disyuntivas del Agro en la Zona Norte de Costa Rica (pp141-158). San José: CIRAD. IIS-UCR.

Rodríguez, N. M. (2013). La etnografía como herramienta en los proyectos de intervención social para el desarrollo. Boletín de Antropología, 27(44), 223-253.

Salazar, M. C. (1992). La investigación-acción participativa: inicios y desarrollos. Madrid: Editorial Popular. 
Sperber, D. (1996). La contagion des idées. Paris: Eds Odile Jacob.

Uribe, C. A. (2005, julio-diciembre). Mímesis y paideia antropológica en Colombia. Revista Antipoda, (1), 67-78.

Uribe, M. V., y Restrepo, E. (2000). Introducción. En Antropologías Transeúntes (pp. 10-22.). Bogotá: Instituto Colombiano de Antropología e Historia.

Valencia, A. (2014). Grandes retos de las ciencias sociales contemporáneas. Revista Colombiana de Sociología, 37(1), 157-175.

Wallerstein, I. (Ed.). (1996). Abrir las ciencias sociales: informe de la Comisión Gulbenkian para la reestructuración de las ciencias sociales. México: Siglo XXI.

White, H. (1973). Metahistory: The Historical Imagination in Nineteenth-Century Europe. Baltimore: The Johns Hopkins University Press.

\section{Cómo citar este artículo}

Rodríguez, N. M. (2016). Características y tipologías de la investigación en ciencias sociales: una reflexión sobre la complementariedad de las funciones de transformar y comprender. Universitas Humanística, 81, 357-385. http:// dx.doi.org/10.11144/Javeriana.uh81.ctic 